\title{
Géolinguistique
}

$14 \mid 2013$

Varia

\section{GéoDialect : « Exploration des outils géomatiques pour le traitement et l'analyse des données géolinguistiques »}

GeoDialect: "Exploration of Geomatics Tools for the Processing and Analysis of Geolinguistic Data"

Silvia Gally, Carole Chauvin, Paule-Annick Davoine, Didier Demolin et Michel Contini

\section{OpenEdition \\ Journals}

Édition électronique

URL : http://journals.openedition.org/geolinguistique/1002

DOI : $10.4000 /$ geolinguistique.1002

ISSN : 2650-8176

Éditeur

UGA Éditions/Université Grenoble Alpes

\section{Édition imprimée}

Date de publication : 15 décembre 2013

Pagination : 187-208

ISBN : 978-2-84310-264-6

ISSN : 0761-9081

\section{Référence électronique}

Silvia Gally, Carole Chauvin, Paule-Annick Davoine, Didier Demolin et Michel Contini, « GéoDialect " Exploration des outils géomatiques pour le traitement et l'analyse des données géolinguistiques » », Géolinguistique [En ligne], 14 | 2013, mis en ligne le 30 avril 2019, consulté le 29 octobre 2020. URL http://journals.openedition.org/geolinguistique/1002; DOI : https://doi.org/10.4000/geolinguistique. 1002 


\title{
GéoDialect : «Exploration des outils géomatiques pour le traitement et l'analyse des données géolinguistiques »
}

\author{
Silvia Gally, Carole Chauvin, Didier Demolin, Michel Contini \\ Université Grenoble Alpes, Gipsa-lab (UMR 5216) \\ Paule-Annick Davoine \\ Université Grenoble Alpes, LIG (UMR 5217)
}

\section{Résumé}

Le projet exploratoire GéoDialect s'intéresse à l'amélioration de la gestion des documents cartographiques utilisés dans le traitement et l'analyse des données géolinguistiques, au moyen de méthodes géomatiques innovantes, par l'utilisation d'un Système d'information géographique (SIG). Plus précisément, nous nous concentrons sur un atlas linguistique - outil de recherche développé en dialectologie - et sur la manière dont nous pouvons transférer et réadapter les données géolinguistiques de leur support initial papier vers un support numérique et informatisé. Nous nous focalisons sur l'Atlas linguistique de la France (Gilliéron et Edmont, 19021910), considéré comme le point de départ de la géolinguistique moderne - 639 points d'enquêtes et 1700 cartes. Il s'agit de développer un traitement cartographique automatique des données qui tient compte des approches et des méthodes adoptées en géolinguistique pour les données lexicales.

\section{Mots-clés}

Géolinguistique, géomatique, cartographie. 


\begin{abstract}
The GeoDialect exploratory project is interested in improving the management of cartographic documents used to process and analyze geolinguistic data. We are concentrating on linguistic atlases, which are an important tool in dialectology. We are also interested in transferring geolinguistic data from paper versions to digital support. The project is mainly focused on the modern Atlas linguistique de la France (Gilliéron and Edmont, 1902-1910), which is considered a starting point of modern geolinguistics. This concerns 639 places of investigation and 1700 maps. The project seeks to develop computerized tools to adapt cartographic processing to the treatment of lexical data.
\end{abstract}

\title{
Keywords
}

Geolinguistics, Geomatics, Cartography.

Le projet exploratoire «Exploration des outils géomatiques pour le traitement et l'analyse des données géolinguistiques », appelé GeoDialect, a été proposé dans le cadre du Labex PERSYVAL ${ }^{1}$. Ce projet fédère deux équipes de recherche de Grenoble : l'équipe Systèmes linguistiques et dialectologie (SLD) du laboratoire GIPSA-lab (UMR 5216) et l'équipe STEAMER du laboratoire LIG (UMR 5217), spécialisée en géomatique. Le projet exploratoire GeoDialect naît de l'intérêt que nous portons à la sauvegarde de la collection de l'Atlas linguistique de la France, actuellement disponible seulement sur support papier, et vise l'exploration des méthodes géomatiques innovantes qui permettent de systématiser le traitement des données géolinguistiques.

L'Atlas linguistique de la France (ALF) a été choisi non seulement pour son état de détérioration avancé sur format papier en raison de son impression datant du début du $\mathrm{XX}^{\mathrm{e}}$ siècle, mais aussi parce qu'il constitue une base de données linguistiques riche en quantité, variété et qualité. À ce jour, les nombreuses données qui composent cet atlas n'ont pas été explorées et analysées dans leur intégralité, ce qui constitue un attrait majeur pour nombreux spécialistes de géolinguistique et d'autres disciplines linguistiques.

L'ALF naît comme le premier grand chantier géolinguistique du début du $\mathrm{Xx}^{\mathrm{e}}$ siècle, sur le lexique des parlers gallo-romans de France, initié par Jules Gilliéron et Edmond Edmont. Entre 1897 et 1901, les auteurs

1. ANR-11-LABX-0025. 
ont effectués des enquêtes de terrain sur 639 communes, au travers d'un questionnaire unique portant à la fois sur la faune, la flore sauvage, les phénomènes météorologiques, l'homme et la vie quotidienne. Les formes lexicales récoltées ont été retranscrites au moyen de l'alphabet phonétique Rousselot-Gilliéron (APG) et représentées sur un support cartographique. Cet atlas compte 1700 cartes - dont 1400 complètes et 300 partielles réunies en 12 volumes et publiées entre 1902 et $1910^{2}$. C'est à partir de cet atlas que Gilliéron fonde la géographie linguistique du $\mathrm{xx}^{\mathrm{e}}$ siècle dont il propose Études de géographie linguistique d'après l'Atlas linguistique de la France (Gilliéron, 1912) et La généalogie des mots qui ont désigné l'abeille (Gilliéron, 1917).

Aujourd'hui l'ALF sert de modèle à de nombreux autres chantiers entrepris par les dialectologues du monde entier : par exemple, les données lexicales de l'atlas sont utilisées pour l'élaboration d'atlas de deuxième génération, dits interprétatifs, tels que l'Atlas Linguarum Europae (19752007) ou l'Atlas linguistique roman (1996-2009). Actuellement, force est de constater qu'en France l'exploration de l'outillage logiciel destiné à exploiter les données géolinguistiques est en retard. Généralement, les dialectologues et les géolinguistes qui travaillent sur des atlas de données brutes effectuent l'analyse et le traitement cartographique de ces mêmes données en deux étapes : une première - effectuée en amont - qui est caractérisée par une analyse manuelle, longue, approfondie et minutieuse des formes relevées dans les atlas; puis une deuxième, qui consiste dans le traitement et la visualisation des données sur un support cartographique afin de représenter la distribution aréale d'un phénomène linguistique donné. De ce fait, les géolinguistes ne s'appuient que rarement sur des outils informatiques destinés à systématiser aussi bien l'analyse que le traitement des données géolinguistiques, exception faite pour les études effectuées en dialectométrie $^{3}$ (Séguy, 1973; Goebl, 2002, 2004, 2008; Aurrekoetxea et

2. Quelques années plus tard, Jules Gilliéron et Edmond Edmont publient des volumes annexes, Les Tables (1912) et Les Suppléments I (1920) où figurent, respectivement, l'index des formes dialectales explorées et les matériaux recueillis en dehors du questionnaire lexical.

3. Nous faisons exclusivement allusion aux travaux géolinguistiques de traitement cartographique de données systématisées (informatisées) en France. Par ailleurs, nous considérons les logiciels PAO tels que Photoshop et Gimp, ou encore des logiciels tels que MapMaker, comme des logiciels de dessin ou de représentation manuelle des données cartographiques - un brillant exemple, dans ce sens, est l'ouvrage de Brun-Trigaud, Le Berre et Le Dû (2005). La situation dans les autres pays d'Europe et d'Amérique du Nord est bien différente et un peu plus avancée en termes de traitement informatisé des données linguistiques : notamment si l'on considère les 
Videgain, 2009). Si en matière d'analyse de données géolinguistiques le travail minutieux du spécialiste reste éminemment manuel - tout comme en termes d'extraction et de (re)transcription des données ${ }^{4}-$ et semble difficilement «automatisable» par une programmation informatique, en revanche le traitement des données pourrait être envisagé de façon systématisée fournissant un appui à la cartographie manuelle souvent peu dotée. Dans ce sens, le projet exploratoire GeoDialect se propose alors de tirer profit des nouveaux logiciels de cartographie et de géomatique pour venir en aide aux «dessins de cartes» couramment pratiqués en dialectologie.

Lorsqu'on s'intéresse à l'ALF, on s'aperçoit que la systématisation du traitement des données devient une question épineuse et centrale, de par la quantité des entrées recensées. L'atlas comporte 1086300 données lexicales (1700 cartes pour 639 localités) fiables, notées de façon homogène, à partir d'un seul questionnaire, avec précisions des lieux, des dates et des circonstances. En partant de ce constat, mais aussi de l'état de vieillissement de l'atlas, le projet se propose de procéder à la construction d'une base de données géolinguistiques associée à la numérisation des cartes et au géocodage des entrées linguistiques de l'ALF, ce qui permettra l'automatisation des traitements cartographiques au moyen d'un Système d'information géographique (SIG).

L'usage de ces outils nécessite de passer par différentes phases : a) la numérisation et le géoréférencement des cartes anciennes; b) la création des couches d'information géographique représentant les données géolinguistiques; c) la structuration et la modélisation des données en vue d'effectuer le traitement lexical ${ }^{5}$.

Le projet GeoDialect définit les spécifications méthodologiques et technologiques d'un SIG pour le traitement des données géolinguistiques et propose des représentations cartographiques adaptées aux besoins des géolinguistes.

Dans cet article, nous présenterons la méthode utilisée pour géocoder les points d'enquêtes de l'ALF et le référencement géographique des entrées lexicales dans un SIG. Ensuite, nous montrerons la manière dont nous avons organisé les données géolinguistiques pour faciliter leur intégration dans

travaux divers et variés de Iannàccaro et Dell'Aquila (2006) - réalisés avec des logiciels développés par les chercheurs -, ceux de Hoch et Hayes (2010), ou encore les travaux de Labov, Ash et Boberg (2006) réalisés sous MapInfo.

4. Se référer aux travaux du THESOC de l'université de Nice : <http://thesaurus.unice. $\mathrm{fr} />$.

5. Dans cet article nous aborderons uniquement la question du traitement des données lexicales par le biais d'un SIG. 
ce type de logiciel. Des exemples et des tests cartographiques effectués dans le cadre du projet montreront l'apport de la géomatique au traitement des données géolinguistiques.

\section{Géocodage des points d'enquêtes de l'Atlas linguistique de la France}

Sur le fond de carte de l'ALF établi pour l'étude des parlers romans de la France et des pays limitrophes, sont exclues les aires/zones géographiques où sont parlées les variétés du basque, du breton, de l'alsacien, du lorrain et certaines localités du département de la Moselle. En revanche, sont pris en compte les parlers wallons de Belgique et du Luxembourg, les parlers gallo-romans d'Italie et de Suisse et les parlers catalans présents sur le territoire français. On peut dénombrer 639 points d'enquête correspondant à 639 localités. Ces points d'enquête sont situés le plus souvent à égale distance les uns des autres, sauf au centre de la France. En effet, dans cette zone la densité de points est moins importante car elle correspond à l'aire de disparition des patois d'oïl.

Dans un Système d'information géographique l'opération de géoréférencement consiste à affecter à une carte numérisée sous la forme d'une image, des coordonnées géographiques en latitude et en longitude, dans un système de référence spatiale. Tout point de la carte géoréférencée a alors une position géographique. A priori, en appliquant cette procédure à la carte de l'ALF, il serait possible d'extraire les coordonnées de chaque point d'enquête défini par Gilliéron. Cependant, ce document publié au début du $\mathrm{XX}^{\mathrm{e}}$ siècle ne possède pas de point d'accroche, comme cela peut être le cas des cartes d'état-major, et la procédure de géoréférencement engendre des déformations, ainsi que des erreurs de localisation et de précision géographique.

En revanche, l'intégration des données géolinguistiques dans un SIG, notamment celles liées à la localisation des points d'enquête, peut s'appuyer sur des procédures de géocodage dont l'objectif est d'associer à un nom de lieu (hameau ou commune), des coordonnées géographiques. Les géocodeurs proposés dans les SIG déterminent les coordonnées d'un lieu ou d'une adresse en utilisant des données géographiques de référence dont les coordonnées sont connues. La précision du géocodage dépend de la qualité des données géographiques de référence. Le Référentiel géographique à grande échelle de l'Institut géographique national, à travers les bases de données Topographique (BD Topo), des Toponymes (BD Nymes) ou Géofla, proposent à différentes échelles des données géographiques sur 
la localisation des communes, les lieux-dits ou des départements. Toutefois, ces noms de lieux de référence sont récents. Or, certaines localités de l'ALF ont pu, soit disparaître depuis 1902, soit changer d'orthographe et/ou changer de nom, ce qui peut induire des erreurs et nécessiter une expertise du domaine. Appliquée aux données de l'ALF, la démarche méthodologique mise en œuvre pour géocoder les points d'enquête s'est déroulée en quatre étapes : 1) création d'un fichier contenant les localités et des numéros des points d'enquête; 2) géocodage proprement dit;3) vérification et affinement du géocodage; 4) validation des résultats. Nous détaillons ci-après l'ensemble de cette procédure.

\subsection{Saisie et géocodage des données de l'Atlas linguistique de la France}

La première étape de la procédure a consisté à saisir dans un tableur (format Excel), la liste des points d'enquête et des localités correspondantes, extraite du volume Tables de l'ALF. Afin de désambiguïser les noms de lieux, en raison des homonymes ou des modifications orthographiques, la table à géocoder est structurée en trois colonnes : le numéro du point d'enquête, le nom de la localité correspondante, et le numéro du département (ou le nom du pays pour la Suisse, la Belgique et l'Italie). Une fois le fichier élaboré, il est importé dans le SIG - dans ce cas le logiciel ArcGIS - où la procédure de géocodage est appliquée. Ce géocodage a été réalisé à partir de deux couches d'information géographique de référence : la couche des communes françaises et la couche des départements - toutes deux issues de la BD Géofla de l'IGN (projection Lambert93). Cette procédure a été réalisée de manière semi-automatique en deux étapes : 1) établissement automatique d'un «matching» ou une correspondance entre les noms des lieux contenus dans la BD Géofla et ceux de la table à géocoder; 2) choix d'une liste potentielle de noms de lieux par l'utilisateur, qui correspondent au mieux lorsque le «matching» ne peut pas être établi. Malgré la lourdeur de cette méthode, les résultats ont été probants puisqu'au total 553 points de l'ALF sur $621^{6}$ ont été codés.

Les points non géocodés du territoire français correspondaient aux noms de communes qui ont été modifiés ou à des hameaux - couche de référence BD Géofla. Une deuxième étape de géocodage a été alors réalisée en utilisant la base de données Toponyme de l'IGN qui contient tous les noms de lieux du territoire français. Une fois les lieux de l'ALF géocodés, leurs

6. Il s'agit, plus précisément, des points linguistiques compris dans le territoire français. 
coordonnées géographiques sont attribuées aux points d'enquête (fig. 1) et représentés sur une carte (fig. 2). Par comparaison entre la table des lieux géocodés et celle des lieux non géocodés, les points d'enquête ne disposant pas de coordonnées géographiques ont été identifiés, puis géocodés par identification manuelle (recherche sur Google).

\begin{tabular}{|r|l|l|l|l|r|r||}
\hline ID * $^{*}$ & Shape * & POINT & \multicolumn{1}{|c|}{ LIEU } & DEPART & \multicolumn{1}{c|}{ X } & \multicolumn{1}{c|}{ Y } \\
\hline 8 & Point & 935 & Surjoux & 01 & 5,799903 & 46,02994 \\
\hline 9 & Point & 913 & Villars-les-Dombes & 01 & 5,045984 & 45,99985 \\
\hline 17 & Point & 776 & Lézignan-Corbières & 11 & 2,765101 & 43,19833 \\
\hline 18 & Point & 784 & Rivel & 11 & 2,005488 & 42,919142 \\
\hline 19 & Point & 785 & Ladern-sur-Lauquet & 11 & 2,392941 & 43,102249 \\
\hline 20 & Point & 786 & Tuchan & 11 & 2,736532 & 42,911309 \\
\hline 21 & Point & 787 & Sigean & 11 & 2,979367 & 43,03428 \\
\hline 22 & Point & 793 & Axat & 11 & 2,242055 & 42,796594 \\
\hline 23 & Point & 773 & Fanjeaux & 11 & 2,035164 & 43,192137 \\
\hline 24 & Point & 718 & Laguiole & 12 & 2,866889 & 44,695653 \\
\hline 25 & Point & 724 & Rieupeyroux & 12 & 2,235421 & 44,303566 \\
\hline 26 & Point & 727 & Espalion & 12 & 2,762465 & 44,519856 \\
\hline 27 & Point & 728 & Séverac-le-Château & 12 & 3,078274 & 44,300124 \\
\hline 28 & Point & 735 & Calmont & 12 & 2,525277 & 44,265039 \\
\hline 29 & Point & 737 & Saint-Rome-de-Tarn & 12 & 2,892253 & 44,03667 \\
\hline 30 & Point & 746 & Belmont-sur-Rance & 12 & 2,751691 & 43,824983 \\
\hline 31 & Point & 748 & Nant & 12 & 3,289054 & 44,02061 \\
\hline 32 & Point & 716 & Conques & 12 & 2,41668 & 44,591572 \\
\hline 33 & Point & 872 & Mlartigues & 13 & 5,047361 & 43,384848 \\
\hline 34 & Point & 873 & Eyguières & 13 & 5,013059 & 43,700592 \\
\hline & & & & & \\
\hline
\end{tabular}

Figure 1. - Extrait de la table géocodée des points d'enquête de l'ALF.

Les colonnes $\mathrm{X}$ et $\mathrm{Y}$ représentent respectivement les coordonnées géographiques en longitude et latitude.

L'ensemble de cette procédure a permis de géocoder $621^{7}$ points de l'ALF. Leur localisation a été validée visuellement en comparant la carte issue du géocodage (fig. 2, page suivante) et la carte originale de l'ALF.

7. Nous ne présentons que la partie des points appartenant à la France. 


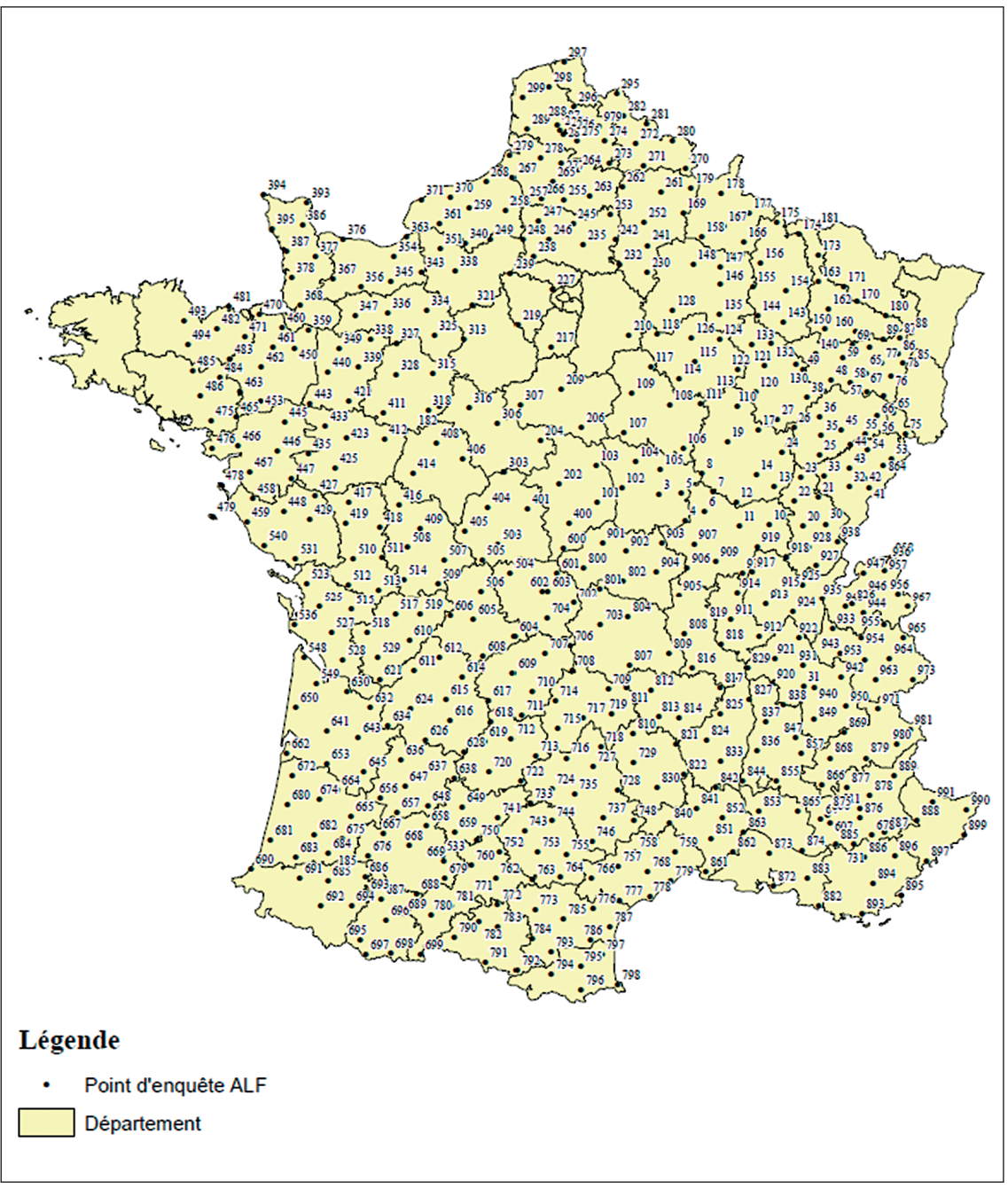

Figure 2. - Géocodage des points d'enquête de I'ALF (C. Cavalière, 2013), d'après J. Gilliéron et E. Edmont, Atlas linguistique de la France, 1912.

\section{Les données lexicales et leur traitement cartographique}

\subsection{Structuration des données pour leur intégration dans un logiciel SIG}

Pour étudier les problématiques liées à une systématisation du traitement cartographique des données géolinguistiques avec un logiciel SIG, les données doivent être structurées et modélisées. 
Le traitement et l'analyse des données lexicales, en géolinguistique, supposent un travail en amont de recherche de lemmatisation, d'étymologie, ou encore de sémantique motivationnelle, qui relève de l'expérience du géolinguiste et de sa propre analyse des données brutes. Du point de vue informatique, la structuration et la modélisation des données lexicales ne peuvent s'effectuer qu'en étroite collaboration avec les experts du domaine et sont fortement liées à leurs besoins, notamment en termes d'analyse.

Compte tenu de ce présupposé, le but de ce projet consiste à chercher et développer des outils informatiques qui, couplés à un Système d'information géographique, facilitent le travail de cartographie que le géolinguiste doit accomplir; des outils qui permettent de visualiser facilement dans un fond de carte la distribution aréale d'un phénomène, ou mieux, d'une désignation linguistique donnée et de voir si cette distribution aréale est caractérisée ou pas, dans un espace géographique où elle se situe.

Pour ce faire, nous nous sommes demandé ce dont les spécialistes en analyse lexicale et motivationnelle ont besoin pour effectuer une étude lorsqu'ils se retrouvent devant les données brutes d'atlas. La réponse étant la forme phonétique, le lemme, l'étymon et la motivation sémantique rattachés à chaque occurrence étudiée.

Les données de l'ALF sont organisées, dans ce sens et dans des tableurs, pour leur exploitation dans un logiciel SIG. Chaque tableau correspond à une désignation et regroupe le nombre d'occurrences contenues dans l'ALF pour cette désignation. Les lignes des tableaux représentent les points linguistiques, les colonnes correspondent à la forme phonétique, au lemme, à l'étymon et à la motivation sémantique. Concernant l'étymon nous avons volontairement rajouté des colonnes «étymon préfixe» et «étymon suffixe» qui renseignent la présence ou l'absence de suffixes ou de préfixes originels associés à l'étymon. Nous avons aussi indiqué le genre des occurrences, par un 'm' pour le masculin et par un ' $\mathrm{f}$ ' pour le féminin, lorsque celui-ci est noté dans l'ALF.

Les figures 3 et 4 (page suivante), montrent l'organisation des données lexicales de l'ALF dans des tableurs - exemples de classement pour les désignations de l'aulne et du champignon. À chaque ligne correspond une forme lexicale, retranscrite en API, reliée à un point linguistique, à un lemme et ainsi de suite. 

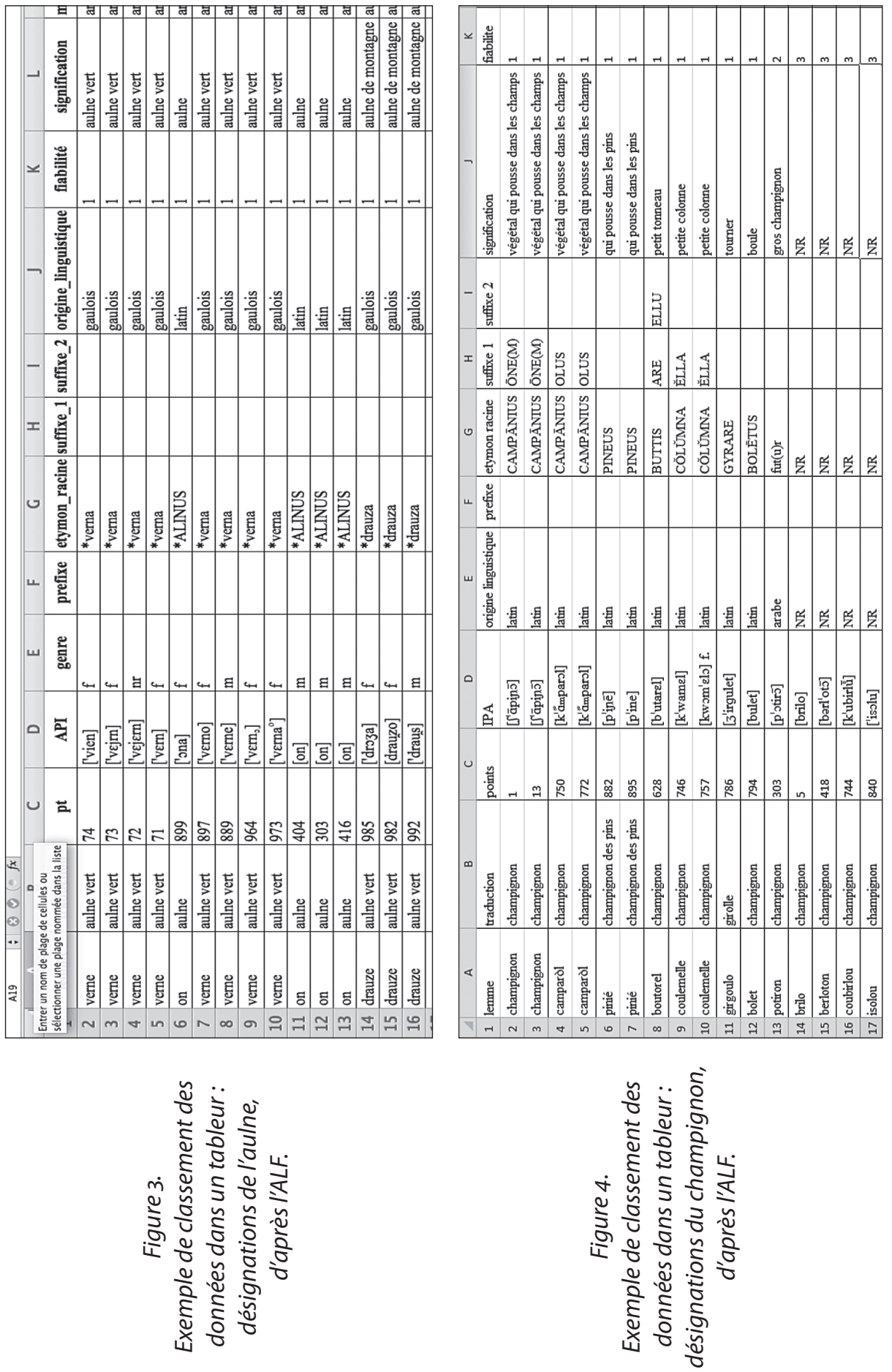
On observe, notamment dans la colonne «IPA», que chaque entrée est enregistrée non pas en alphabet phonétique Rousselot-Gilliéron (APG), mais en Alphabet phonétique international (API). Les correspondances entre alphabets APG et API ont soulevés différentes problématiques que nous avons traitées au cas par cas, notamment pour le groupe de données ${ }^{8}$ que nous explorons dans ce projet.

Dans la dernière colonne de ces tableaux - nommée «fiabilité» nous avons aussi pris le soin de noter un degré de fiabilité pour chaque forme lexicale examinée par le spécialiste : cet indice, allant de 1 à 3 , respectivement d'un «maximum de fiabilité» à un «minimum de fiabilité» évalue la fiabilité des reconstructions étymologiques et/ou motivationnelles effectuées pour chaque forme, par le géolinguiste ${ }^{9}$. Ceci nous permet d'évaluer la pertinence de l'analyse menée par les géolinguistes sur n'importe quelle occurrence, outre de favoriser un repérage rapide par rapport à l'ensemble des données. En outre, le sigle 'nr' - «non renseigné» permet de classer tout manque d'information relatif à un élément (colonne) de l'entrée lexicale ${ }^{10}$. Le but étant, encore une fois, de faciliter le repérage des formes, ainsi que leur classement, à l'intérieur d'un tableur de plus de 600 lignes multiplié par une dizaine de colonnes. Par ailleurs, ces critères de notations répondent à des techniques de classement adoptées dans la constitution de bases de données informatisées, ainsi qu'en géomatique dans un SIG.

8. Les cartes de l'ALF que nous avons sélectionnées sont celles de l'aulne, du bleuet, du bouleau, du champignon, du châtaignier, du chêne, du coquelicot, de l'églantier, de l'érable, du genévrier, de l'hêtre, du pissenlit, du plantain; sans oublier la carte du chat et celle du cheval.

9. Les spécialistes ont parfois des difficultés à classer une ou plusieurs formes au sein d'un groupe de désignations : ces difficultés peuvent être liées aux documentations scientifiques consultées pour l'analyse des formes. Il arrive que les spécialistes en analyse lexicale n'aient pas un avis définitif et tranché sur l'entrée linguistique examinée : par exemple, ces problématiques peuvent concerner la reconstruction sémantique (motivationnelle) et/ou étymologique qui s'avère être faiblement (ou pas) documentée, ou bien documentée de façon différente, selon la source bibliographique à laquelle on fait référence. De manière générale, les indices de fiabilités tiennent compte des sources scientifiques sur lesquelles le spécialiste s'appuie pour effectuer une analyse lexicale, annotées dans les tableurs sous la section «commentaires».

10. Il peut s'agir d'un manque d'informations de différentes natures touchant soit au «genre» de la forme - comme le montre la figure 3 -, soit à la reconstruction étymologique ou motivationnelle qui peuvent demeurer inexplicables - lignes 14 à 17 , figure 4 . 
Concernant les formes lexicales notées en APG dans l'ALF, nous avons maintenu les notations des caractères en indice ou en exposant dans leur retranscription en API ${ }^{11}$, pour éviter les problématiques liées à la réinterprétation et la retranscription des données - notamment dans le cadre des notations sur les degrés d'aperture ou de fermeture des voyelles de l'ALF. Par ailleurs, nous avons convenu de retranscrire les formes en API sans toucher à l'accentuation tonique gilliéronienne ${ }^{12}$ : les formes inaccentuées ont été aussi retranscrites telles quelles en API, en sachant que l'absence d'accent tonique est le témoin d'une accentuation en syllabe finale du mot - comme en français.

Aussi, nous avons limité les problématiques liées aux notations des diphtongues et triphtongues en adoptant les mêmes règles de retranscription phonétiques utilisées dans l'Atlas linguistique roman. Notamment, nous avons choisi de retranscrire les diphtongues descendantes en API avec un diacritique, comme par exemple [au], et de noter les diphtongues ascendantes en employant les approximantes, comme dans les cas [wo] et [je].

Nous nous limitons à l'exposition de ces exemples précis, sans rentrer dans les débats et les problématiques bien connus au sujet de la nature des données de l'ALF et des retranscriptions en APG - notamment dans le cas des voyelles.

\subsection{De la structuration des données à leur modélisation}

Du point de vue informatique, ce travail de structuration et de formalisation des données géolinguistiques a permis l'élaboration d'un modèle de données. Un tel modèle présente l'intérêt de permettre l'intégration dans un SIG, de façon systématique et homogène des données de l'ALF, au fur et à mesure des besoins et des études menées par les géolinguistes. La figure 5 présente la façon dont les données doivent être formalisées. Dans ce modèle, les éléments qui constituent les tableaux de données décrits ci-dessus sont regroupés sous l'entité (eNTRÉE LINGUISTIQUE); d'autres éléments tels que la description des données (MÉTADOnNÉEs) qui se présentent sous la forme d'un commentaire, ainsi que les cartes (CARTES) associées aux points d'enquête (POINT ALF) et qui sont regroupées dans les fascicules (FASCICULE) des différents volumes de l'ALF (vOLume). Les cartes représen-

11. Cf. lignes 9 et 10 du tableur dans la figure 3 .

12. Exception faite pour la longueur et la brièveté des voyelles qui n'ont pas été retenues dans la retranscription en API. 
tant les entrées linguistiques sont numérisées pour être stockées et consultées dans le SIG sous forme d'images (éventuellement géoréférencées).

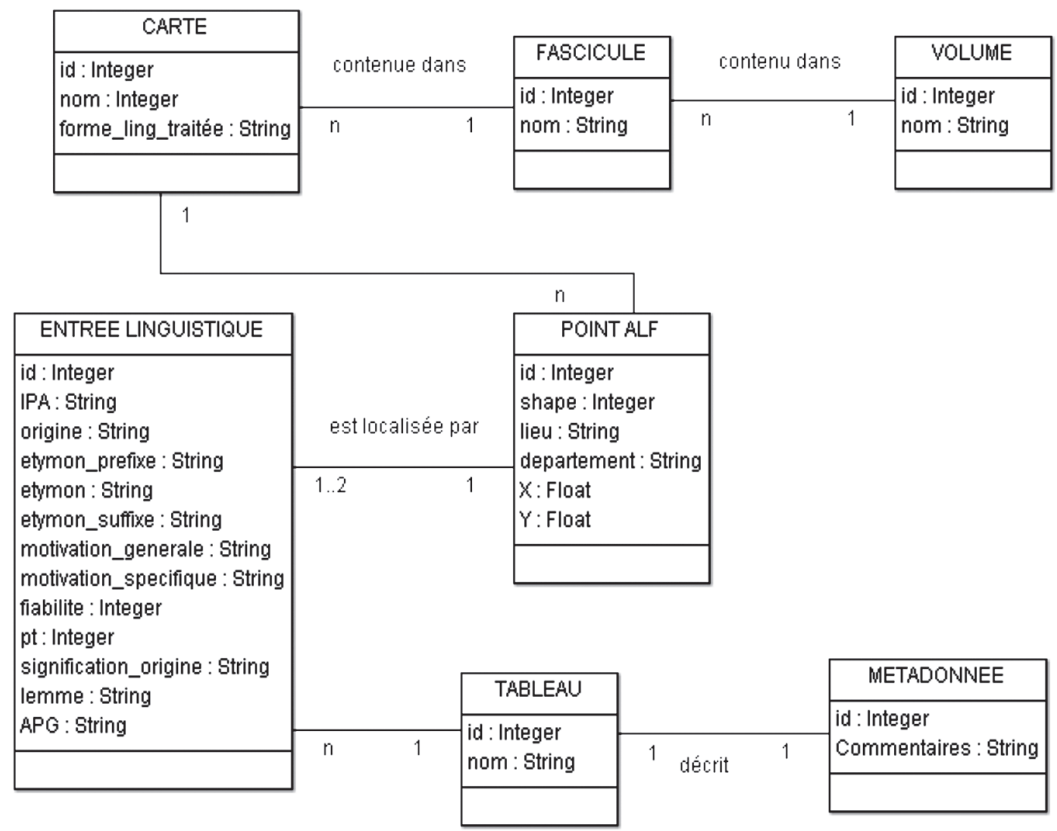

Figure 5. - Modèle de données géolinguistiques pour l'étude des données de l'ALF.

\subsection{Propositions cartographiques des données géolinguistiques}

Après avoir compilé et renseigné les tableaux manuellement - analyses lexicale et motivationnelle - les données structurées dans les tableurs (les formats peuvent être csv ou txt) sont intégrées dans le logiciel SIG au moyen d'une jointure entre les points linguistiques géocodés et informations lexicales associés à ces points : à chaque point linguistique correspond un certain nombre d'informations sur les occurrences lexicales (forme phonétique, lemme, étymon, etc.).

Dans les cartes ci-après, nous proposons, à partir d'un jeu de données test correspondant aux désignations de la «toupie» et du «champignon», différentes représentations cartographiques.

La figure 6 a pour objectif de représenter la répartition des formes phonétiques et de leurs désignations selon la distribution des points d'enquête. À chaque forme est associée une couleur spécifique permettant leur différenciation. La lecture de ce simple test permet déjà un premier niveau d'interprétation par identification des jeux de couleur. 


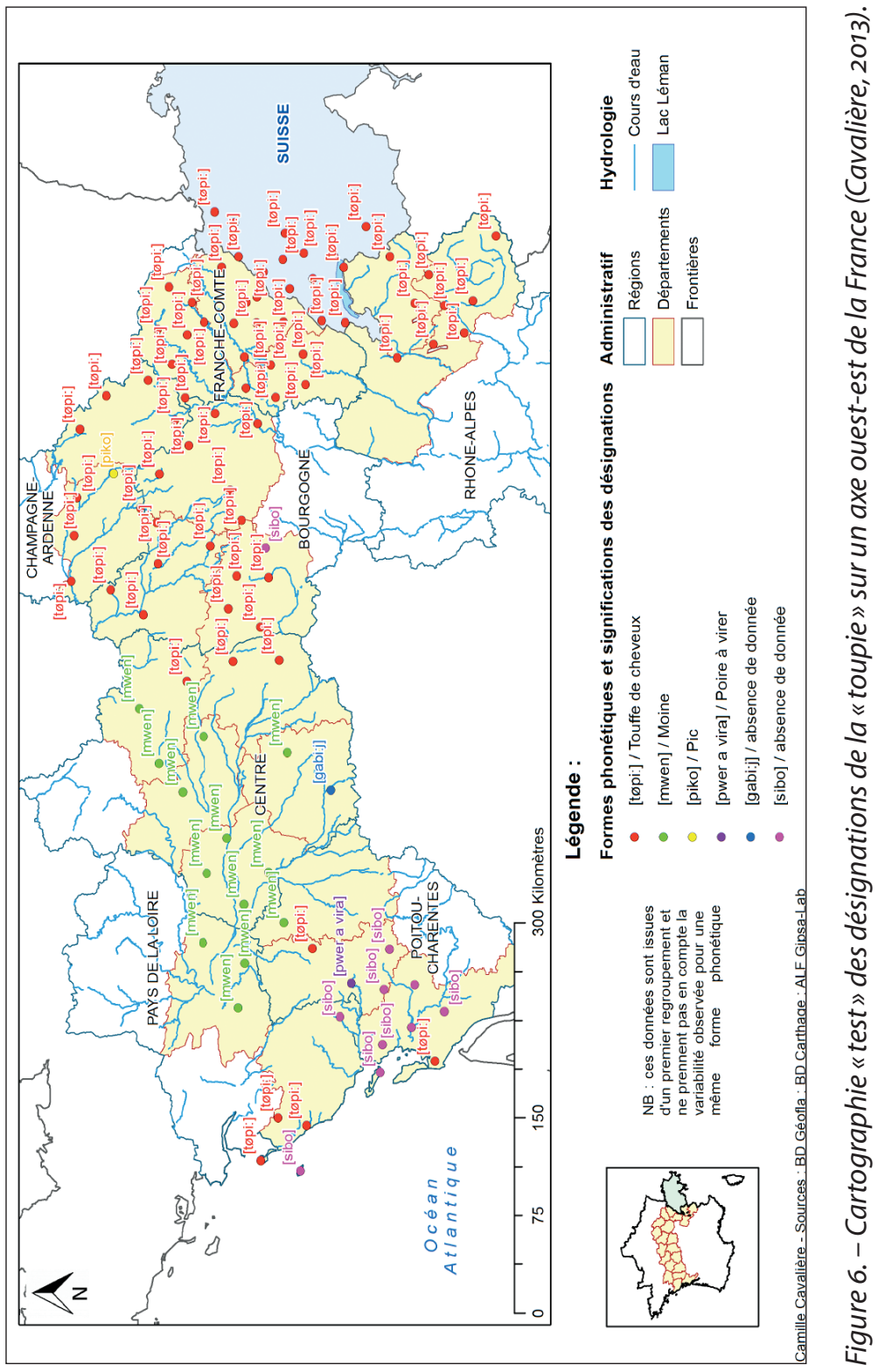


Dans un second temps, nous avons voulu proposer une représentation cartographique en isoglosses. Pour cela nous avons utilisé des fonctionnalités d'analyse spatiale proposées par le SIG ArcGIS, notamment celles relatives à la construction de polygones de Thiessen ${ }^{13}$. Cette méthode utilisée en dialectométrie (Séguy, 1973; Goebl, 2002, 2004, 2008) consiste à établir une triangulation (triangulation de Delaunay) qui relie chaque point d'enquête situé dans un espace géographique avec ceux de son voisinage. Les polygones sont construits en considérant la médiatrice des arêtes entre deux points, ce qui permet de construire un maillage du territoire. Dans notre contexte, chaque maille représente alors l'espace géographique théorique couvert par un point d'enquête (fig. 7). La figure 7 représente donc la construction de ces polygones effectués sur l'espace géographique couvert par les points d'enquête de l'ALF.

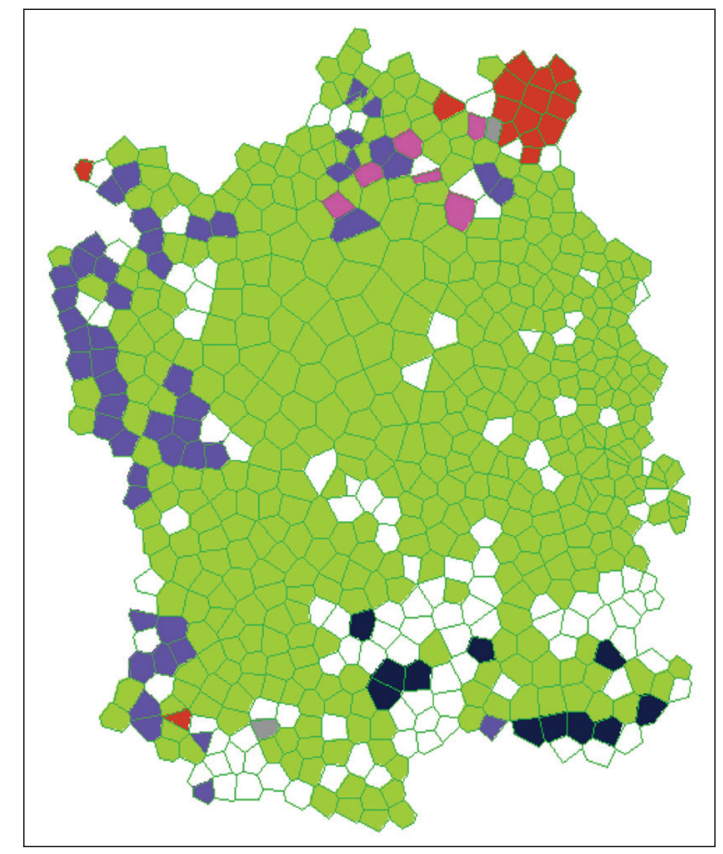

Figure 7. - Exemple de regroupement par «lemmes» pour les désignations de la «toupie».

Maillage des points d'enquête de l'ALF réalisé selon la méthode des polygones de Thiessen.

13. À ce propos, voir <http://resources.arcgis.com/fr/help/main/10.1/index.html\#//000 $80000001 \mathrm{~m} 000000>$. 
Ce maillage constitue donc la base du processus de construction des isoglosses qui définissent et délimitent les aires géolinguistiques selon différents regroupements des formes : que ce soit par lemme ou bien par étymon ou par forme phonétique. Au moyen d'un SIG et pour chaque entrée linguistique étudiée, ce processus se décline en plusieurs étapes :

1) jointure attributaire entre les points d'enquête géocodés et les désignations associées aux points d'enquête. Cette première étape permet de créer la couche d'information géographique des désignations et d'obtenir leur répartition géographique. La figure 8 (carte du haut) montre, par exemple, le traitement effectué sur un extrait du jeu de données «toupie»;

2) superposition de la couche d'information géographique des désignations et de celle du maillage des points d'enquête (polygone de Theissen). À partir de cette superposition et au moyen d'une jointure spatiale entre ces deux couches, il est possible d'affecter à chaque maille (ou polygone) la valeur de la désignation associée au point d'enquête. On obtient alors une carte en aplat de la répartition des désignations ou des paramètres en fonction non plus des points mais des mailles (fig. 7);

3) agrégation des mailles en fonction de leur valeur et de leur position les unes par rapport aux autres. Cette opération nous permet de définit des aires géographiques homogènes et lissées (fig. 8, carte du bas).

D'autres méthodes d'analyse spatiale, comme la méthode des voisins naturels ont aussi été testées sous ArcGIS (fig. 9). Toutefois, cette méthode - s'appliquant essentiellement sur des données quantitatives - n'a pas été retenue. 

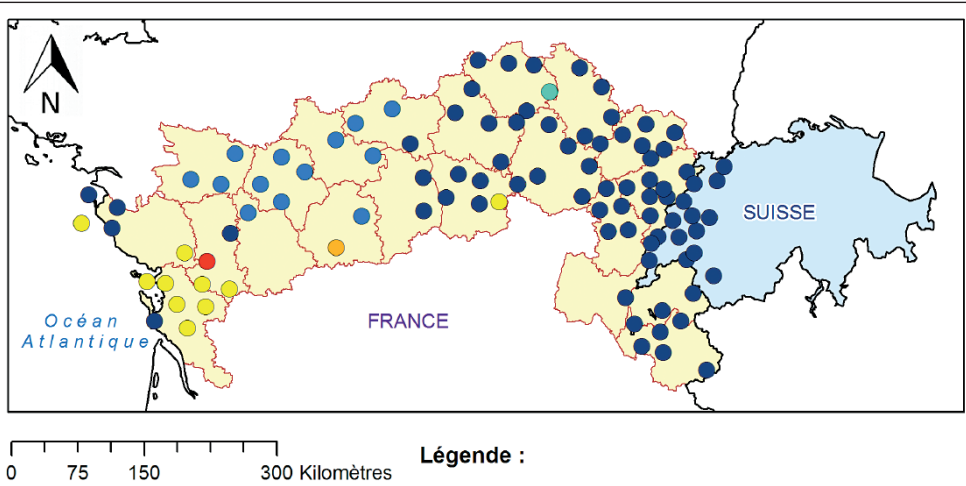

\section{Légende :}

Motivation spécifique

Administratif

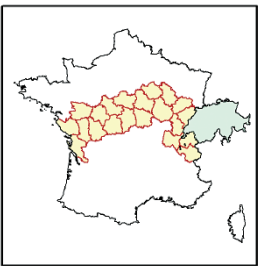

non renseigné

- Aspect arrondi, ventru; bedaine

$\square$ Limites de départements

- Forme, mouvement

- Pointe, clou

- Sommet, tête

Tête d'oignon
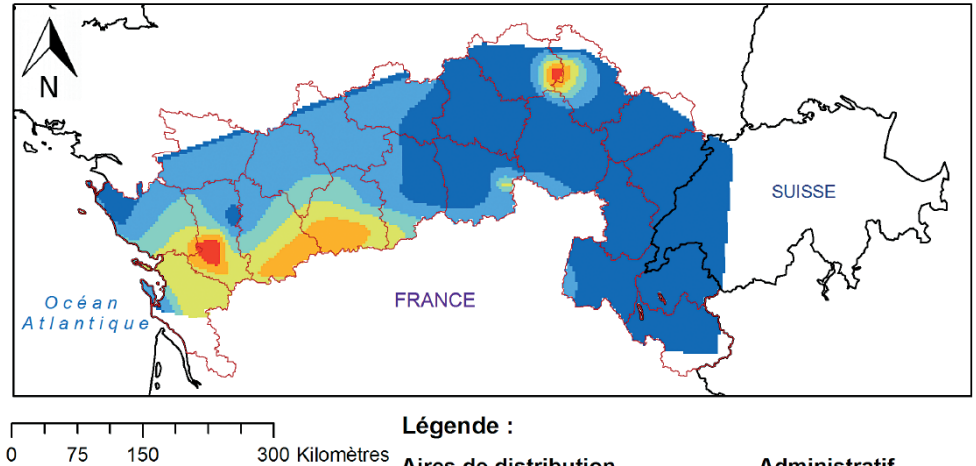

Légende :

$\begin{array}{lll}75 & 150 & 300 \text { Kilomètres }\end{array}$

Aires de distribution

Interpolation réalisée par la méthode des voisins naturels

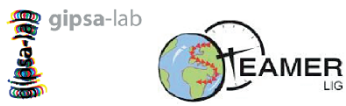

\section{Administratif}

Limites de départements

Forme, mouvement

non renseigné

Tête d'oignon

Pointe, clou

Aspect arrondi, ventru, bedaine

Sommet, tête

Figure 8. - Deux types de représentation des critères motivationnels dans les désignations de la «toupie» pour le centre de la France.

Extrait de Cavalière (2013). 


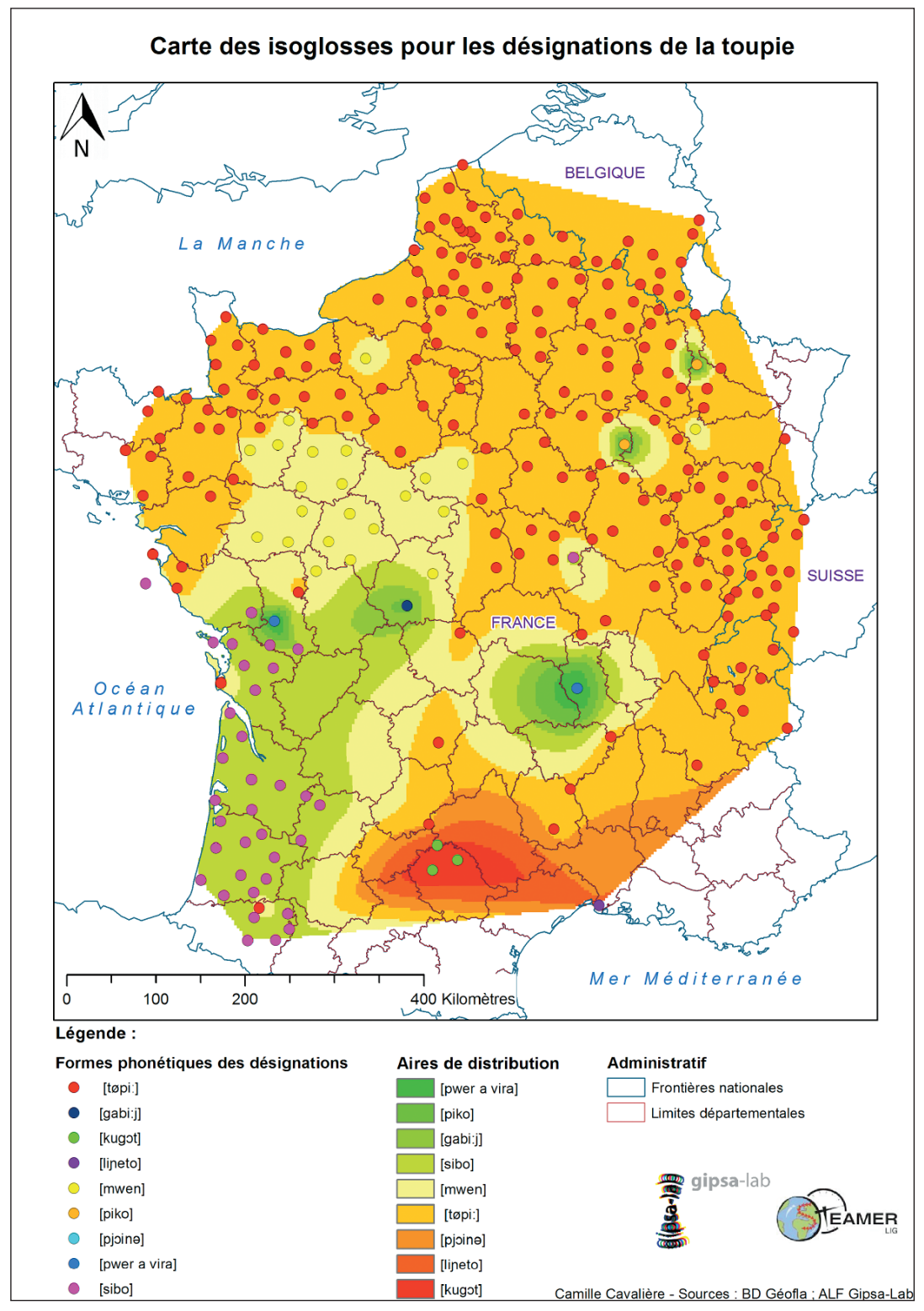

Figure 9. - Test de carte en aires lexicales, pour la désignation de la «toupie». Extrait de Cavalière (2013). 
Le processus de création d'isoglosses basées sur la méthode des polygones de Thiessen étant relativement lourd et répétitif, nous avons proposé de l'automatiser en créant dans ArcGIS un outil spécifique (outil «Créer des isoglosses»). L'intérêt de cette proposition est que le processus devienne transparent à l'utilisateur pour que ce dernier puisse choisir de cartographier en regroupant les formes linguistiques par lemme, par forme phonétique ou par étymon (fig. 10).

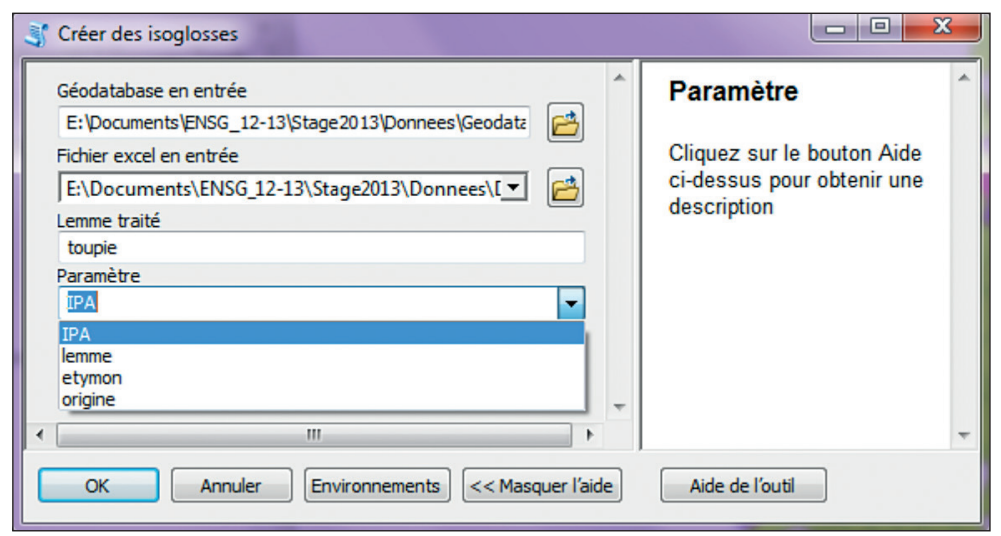

Figure 10. - Interface de l'outil création d'isoglosses sous ArcGIS.

L'utilisation de cet outil est particulièrement simple : l'utilisateur saisit le nom du dossier contenant le fichier de données; il renseigne le nom du fichier Excel dans lequel sont stockées les données linguistiques structurées selon le format défini au paragraphe 2.1 ; il renseigne la désignation à traiter (ici, «toupie»), puis sélectionne un paramètre de son choix (lemme, forme API, ou étymon). Une fois validé, le traitement est exécuté immédiatement. Une nouvelle couche d'information géographique contenant les contours des isoglosses est générée. L'utilisateur peut à son gré visualiser et apporter des modifications de graphismes (choix de couleurs) sur son document cartographique.

Cette proposition a été développée sous ArcGIS, mais des modifications ultérieures sont actuellement en cours pour les transposer sur le logiciel SIG QGIS. Par ailleurs, nous souhaitons tester et implémenter d'autres méthodes de construction d'isoglosses en nous appuyant sur des méthodes d'interpolation appliquées aux données qualitatives. 


\section{Conclusions}

Dans cet article, nous avons exposé les travaux effectués dans le projet GeoDialect - toujours en cours de réalisation - qui s'intéressent à la cartographie des données géolinguistiques brutes, issues de l'Atlas linguistique de la France. Nous pensons qu'un processus d'automatisation cartographique des données lexicales par le biais d'un SIG, comme celui que nous sommes en train de mettre en place, se construit sur le travail analytique préliminaire effectué par le spécialiste - le géolinguiste. L'approche géolinguistique et l'approche informatique (géomatique) adoptées dans le cadre de ce projet tiennent compte de l'expérience et du savoir faire des membres de l'équipe SLD ${ }^{14}$ spécialisés dans le traitement et dans l'analyse géolinguistiques des données lexicales, mais aussi dans les théories et approches plus récentes de la géolinguistique motivationnelle.

D'un point de vue informatique, ces premières explorations ont montré que les outils SIG pouvaient contribuer à l'amélioration des traitements des données géolinguistiques et favoriser leur analyse. Toutefois, ils ont aussi obligé à structurer les données géolinguistiques de façon très rigoureuse, afin d'éviter tout problème lors de leur intégration dans les SIG.

Ces explorations doivent se poursuivre, aussi bien dans une "phase amont» pour la construction des tableaux de données en proposant des outils d'aide à la saisie et au traitement des données pour l'intégration dans un SIG - en se concentrant sur la lemmatisation, par exemple - , que dans une «phase aval» pour la définition des formes de représentation cartographique innovantes, comme la représentation en 3D.

\section{Remerciements}

Ce projet a été financé par le Labex PERSYVAL, ANR-11-LABX-0025. Nous remercions Camille Cavalière, étudiante de l'Institut de géographie alpine (IGA), et Camille Briend, étudiante de l'École normale des sciences géographiques (ENSG), pour leur participation au projet et leurs travaux.

14. La formation de l'équipe SLD suit l'intégration de l'ancien Centre de dialectologie de Grenoble dans le GIPSA-lab en 2008. 


\section{RÉFÉRENCES BIBLIOGRAPHIQUES}

ALE - Atlas Linguarum Europae, 1975-2007, Assen, Van Gorcum.

ALiR - Atlas linguistique roman, 1996-2009, Rome, Istituto Poligrafico e Zecca dello Stato.

Aurrekoetxea Gotzon et Videgain Charles, 2009, «Le projet Bourciez : traitement géolinguistique d'un corpus dialectal de $1885 »$, Dialectologia, $\mathrm{n}^{\circ} 2$, p. 81-111. Disponible sur <www.publicacions.ub.edu/revistes/dialectologia2/> (consulté entre novembre 2012 et janvier 2013).

BRIEND Camille, 2013, Intégration de données dans un SIG, Rapport de stage sous la direction de P.-A. Davoine, Grenoble, Université Joseph Fourier.

Brun-Trigaud Guylaine, Le Berre Yves et Le Dû Jean, 2005, Lectures de l'Atlas linguistique de la France de Gilliéron et Edmont. Du temps dans l'espace, Paris, Éditions du Comité des travaux historiques et scientifiques.

CAvalière Camille, 2013, Géolinguistique et cartographie d'inondations historiques en France, Rapport de stage sous la direction de P.-A. Davoine, Grenoble, Université Joseph Fourier.

DauZAT Albert, 1922, La géographie linguistique, Paris, Flammarion.

Gilliéron Jules, 1912, Études de géographie linguistique d'après l'Atlas linguistique de la France, Paris, Champion.

GiLliéron Jules, 1917, La généalogie des mots qui ont désigné l’abeille, Paris, Champion.

Gilliéron Jules et Edmont Edmond, 1902-1910, Atlas linguistique de la France, 35 fascicules, Paris, Champion.

Gilliéron Jules et Edmont Edmond, 1902, Atlas linguistique de la France. Notice servant à l'intelligence des cartes, Paris, Champion.

Gilliéron Jules et Edmont Edmond, 1912, Atlas linguistique de la France. Tables, Paris, Champion.

Gilliéron Jules et Edmont Edmond, 1914, Atlas linguistique de la Corse, Paris, Champion.

Gilliéron Jules et Edmont Edmond, 1920, Atlas linguistique de la France. Suppléments I, Paris, Champion.

Glaser Elvira et BART Gabriela, 2011, «Discovering and Mapping Syntactic Areas: Old and New Methods», dans X. A. Perez Alvarez, E. Carrilho et C. Magro (éds), Proceedings of the International Symposium on Limits and Areas in Dialectology (LimiAr), Lisbonne, Centro de Linguistica da Universidade de Lisboa, p. 345-363.

Goebl Hans, 2002, «Analyse dialectométrique des structures de profondeur de l'ALF», Revue de linguistique romane, $\mathrm{n}^{\circ}$ 66, p. 5-63.

Goebl Hans, 2004, «Bref aperçu sur les problèmes et méthodes de la dialectométrie (avec application à l'ALF)», dans J.-M. Eloy (éd.), Des langues collatérales : problèmes linguistiques sociolinguistiques et glottopolitiques de la proximité linguistique, vol. 1, Paris, L'Harmattan, p. 39-60.

Goebl Hans, 2008, «La dialettometrizzazione integrale dell'AIS. Presentazione dei primi risultati », Revue de linguistique romane, $\mathrm{n}^{\circ}$ 72, p. 25-113. 
Hoch Shawn et Hayes James J., 2010, «Geolinguistics: The Incorporation of Geographic Information Systems and Science», The Geographical Bulletin, $\mathrm{n}^{\circ}$ 51, p. 23-36.

Iannàccaro Gabriele et Dell'Aquila Vittorio, 2006, Survey ladins. Usi linguistici nelle valli ladine, Trente, Regione Autonoma Trentino-Alto Adige/Südtirol.

Labov William, Ash Sharon et Boberg Charles, 2006, The Atlas of North American English: Phonetics, Phonology and Sound Change, Berlin, Mouton de Gruyter.

Nerbonne John, 2010, «Mapping Aggregate Variation», dans A. Lameli, N. R. Kehrerin et S. Rabanus (éds), Language and Space. Vol. 2 : Language Mapping, Groningen, Mouton de Gruyter, p. 476-495.

SÉGUY Jean, 1973, «La dialectométrie dans l'Atlas linguistique de la Gascogne», Revue de linguistique romane, $\mathrm{n}^{\circ} 37, \mathrm{p} .1-24$. 